\title{
Magnetic excitations near the quantum phase transition in the Ising ferromagnet $\mathrm{LiHoF}_{4}$
}

\author{
H. M. Rønnow, ${ }^{1,2}$ J. Jensen, ${ }^{3}$ R. Parthasarathy, ${ }^{4}$ G. Aeppli, ${ }^{5}$ T. F. Rosenbaum, ${ }^{4}$ D. F. McMorrow, ${ }^{5}$ and C. Kraemer ${ }^{2,1}$ \\ ${ }^{1}$ Laboratory for Quantum Magnetism, École Polytechnique Fédérale de Lausanne (EPFL), Lausanne, Switzerland \\ ${ }^{2}$ Laboratory for Neutron Scattering, ETH-Zürich and Paul Scherrer Institute, 5232 Villigen, Switzerland \\ ${ }^{3}$ Niels Bohr Institute, Universitetsparken 5, 2100 Copenhagen, Denmark \\ ${ }^{4}$ The James Franck Institute and Department of Physics, The University of Chicago, Illinois 60637, USA \\ ${ }^{5}$ London Centre for Nanotechnology and Department of Physics and Astronomy, UCL, London, WC1E 6BT, United Kingdom
}

(Received 4 April 2006; revised manuscript received 19 October 2006; published 28 February 2007)

\begin{abstract}
Effective-medium theory and inelastic neutron scattering are used to study the magnetic excitations in the Ising ferromagnet $\mathrm{LiHoF}_{4}$ near its magnetic-field-induced quantum phase transition (QPT). As expected, the dominant mode softens at the QPT, but reaches a finite, rather than vanishing, limit due to the hyperfine interaction. The experimental phase diagram and excitation spectra are well described by an effective-medium theory to first order in the $1 / z$ expansion. There are some differences between theory and experiments, which may be due to domain walls, their dynamics and shape effects, and to magnetoelastic couplings.
\end{abstract}

DOI: 10.1103/PhysRevB.75.054426

PACS number(s): 75.10.Jm, 75.40.Gb, 75.50.Dd

\section{INTRODUCTION}

Recent years have witnessed considerable interest in quantum phase transitions (QPTs), in which quantum fluctuations as a function of some control parameter such as magnetic field, pressure, or doping drive a system from one ground state to another. ${ }^{1}$ Owing to its simplicity, one of the most studied quantum critical models is the Ising ferromagnet in a transverse magnetic field. ${ }^{1-4}$ Here we describe the experimental model developed to interpret a neutron scattering investigation ${ }^{8}$ of the excitation spectrum in $\mathrm{LiHoF}_{4}$, which is a physical realization of the transverse-field Ising model. ${ }^{5-7}$

$\mathrm{LiHoF}_{4}$ adopts a Scheelite lattice $(a=5.175 \AA$ and $c$ $=10.75 \AA$ ), such that the crystal-field ground state of each Ho ion is a $\Gamma_{3,4}$ doublet, for which only the $c$ component of the angular momentum is nonzero. At an energy of $11 \mathrm{~K}$ above the ground state, the lowest excited crystal-field level is unpopulated at low temperatures. A transverse field (in the $a-b$ plane) mixes higher levels into the ground state, thereby splitting the doublet, with the result that at low temperatures the behavior is that of the transverse-field Ising model. Mixing the two degenerate crystal field states of each Ho ion by a transverse field (whether applied externally or generated internally by the off-diagonal part of the dipole interactions) is the key ingredient for several interesting collective quantum effects in the dilution series $\mathrm{LiHo}_{x} \mathrm{Y}_{1-x} \mathrm{~F}_{4}$, including tunneling of single moments and domain walls, quantum annealing, entanglement, and Rabi oscillations. ${ }^{9,10}$ The work of Giraud et al. ${ }^{9}$ demonstrated the importance of coupling between $4 f$ electrons and nuclear spins of individual Ho ions ( $x=0.002$ in the dilution series). In the case of interacting Ho ions $(x=1)$ the hyperfine interaction introduces new features into the phase diagram, and the nuclear spin degrees of freedom strongly affect the dynamical behavior near the quantum critical point. ${ }^{8}$

The magnetic interaction between the Ho ions is weak and is dominated by the classical dipole-dipole interaction. At zero field $\mathrm{LiHoF}_{4}$ orders ferromagnetically at $T_{C}$ $=1.53 \mathrm{~K}$. When a transverse field is applied the ordering temperature is reduced and approaches zero at a field of 50 kOe. The magnetic phase diagram determined from susceptibility measurements by Bitko et al. ${ }^{6}$ was analyzed in terms of a mean-field (MF) model based on the crystal-field parameters determined by Hansen et al. ${ }^{11}$ and the known hyperfine interaction for holmium. Here we present an effective-medium analysis of both the magnetic phase diagram and the excitation spectrum that goes beyond the MFrandom-phase approximation (RPA). Instead of using the crystal-field parameters of Hansen et al. we derive a new set, which combines their results with those obtained from subsequent spectroscopic investigations of the crystal-field levels of $\mathrm{Ho}$ in $\mathrm{LiYF}_{4} .{ }^{12-14}$ The leading-order effects due to the fluctuations are included by utilizing the high-density $1 / z$ expansion $^{3}$ within the effective-medium approach, ${ }^{15,16}$ and the calculated phase diagram is compared with that obtained from a quantum Monte Carlo calculation of Chakraborty et $a l .{ }^{17}$ The $1 / z$ expansion theory is nearly identical to the one applied in the case of the singlet-singlet system $\mathrm{HoF}_{3},{ }^{18}$ which has a close resemblance to the present system. ${ }^{19,20}$

\section{THEORY}

\section{A. Refinement of the Hamiltonian}

The $S_{4}$ point symmetry of the Ho-ion surroundings in the Scheelite lattice of $\mathrm{LiHoF}_{4}$ implies that the crystal-field Hamiltonian may be written

$$
\mathcal{H}_{\mathrm{CF}}=\sum_{l=2,4,6} B_{l 0} O_{l}^{0}+\sum_{l=4,6} B_{l}^{4}(c) O_{l}^{4}(c)+B_{6}^{4}(s) O_{6}^{4}(s)
$$

where $O_{l}^{m}$ are the Stevens' operators defined, for instance, in Ref. 16. The $z$ axis of the Hamiltonian is along the $c$ axis, whereas the $x$ axis (modulo $\pi / 2$ ) is determined by the requirement that $B_{4}^{4}(s)$ vanishes and $B_{4}^{4}(c)$ is positive. In the experiments referred to below, the transverse field is along the crystallographic [100] direction. The crystal plane of Ho ions, which is perpendicular to the $a$ axis, is a mirror plane for the $\mathrm{Li}$ and $\mathrm{Ho}$ ions, but not for the $\mathrm{F}$ ions. Instead, the fluoride ions have a choice between two equivalent configu- 
TABLE I. Crystal-field parameters in units of meV. The first row contains the parameters from Ref. 11. The two next rows show the results obtained from the optical spectrum of diluted Ho ions in $\mathrm{LiYF}_{4}$. The last row shows the parameters derived in the present work. The set of parameters are the same for all four Ho sublattices. The sign of $B_{6}^{4}(s)$ is undetermined, not arbitrary, and depends on the chosen fluoride basis.

\begin{tabular}{lcccccc}
\hline \hline Ref. & $B_{2}^{0}$ & $10^{3} B_{4}^{0}$ & $10^{3} B_{4}^{4}$ & $10^{5} B_{6}^{0}$ & $10^{5} B_{6}^{4}(c)$ & $10^{5} B_{6}^{4}(s)$ \\
\hline 11 & -0.065 & 0.426 & 4.53 & 0.01 & 8.55 & \pm 1.69 \\
22 & -0.052 & 0.281 & 3.70 & 0.07 & 7.04 & 0 \\
23 & -0.056 & 0.325 & 3.61 & 0.02 & 7.58 & 0 \\
& -0.06 & 0.35 & 3.6 & 0.04 & 7.0 & \pm 0.98 \\
\hline \hline
\end{tabular}

rations, where one is derived from the other by a reflection in this plane. This reflection changes the sign of $B_{6}^{4}(s)$. In a one-domain sample, a point-charge calculation suggests that $B_{6}^{4}(s)$ is positive and that the $x$ axis makes an angle of about $-11^{\circ}$ with [100]. Here we use the usual definitions of the $a$ and $b$ axes and the $\mathrm{F}$ ions are placed at the positions given in, for instance, Ref. 21. The angle is small and assumed to be zero in the following calculations. The sign of $B_{6}^{4}(s)$ has no effect on the present model calculations and is left undetermined. Hansen et al. derived a set of crystal-field parameters exclusively from their susceptibility measurements. ${ }^{11}$ Since then a number of spectroscopic measurements has provided energies of several of the crystal-field levels. ${ }^{12-14}$ Table I shows the parameters of Hansen et al. and those obtained from spectroscopy investigations of dilute systems, ${ }^{22,23}$ in comparison with the ones derived in the present analysis. The parameters of Hansen et al. optimize the fitting to their susceptibility measurements, but the present ones do not change the susceptibility components much. The only discrepancy found in the present fit is that the $c$-axis susceptibility is slightly larger (at maximum 3\%) than measured at

TABLE II. Relative energies in K of the crystal-field levels. The experimental results shown in the three columns to the right are compared with results obtained from the present fit and those predicted by the parameters of Hansen et al. (Ref. 11). The ground state and levels marked by an asterisk are doublets.

\begin{tabular}{lcccc}
\hline \hline This work & Ref. 11 & Ref. 12 & Ref. 13 & Ref. 14 \\
\hline 11 & 9 & 11 & 12 & \\
32 & 39 & 33 & 37 & \\
72 & 74 & & 71 & \\
84 & 92 & & 88 & 85 \\
$109 *$ & 124 & & 112 & 102 \\
328 & 398 & & & \\
404 & 492 & & & \\
$410 *$ & 497 & & & 407 \\
420 & 511 & & & \\
438 & 535 & & & \\
$443 *$ & 546 & & & \\
469 & 575 & & & \\
\hline \hline
\end{tabular}

intermediate temperatures. Most importantly, the present set of parameters describes accurately the $c$-axis field dependence of the three lowest crystal-field levels as measured with electron paramagnetic resonance. ${ }^{12}$ The calculated value of the longitudinal $g$ factor, $g_{\|}=13.78$, is accounting for the saturation value of the $c$-axis moment shown in Fig. 3 of Ref. 11 , and it is equal to the weighted average of this parameter determined from various experiments (see Table II in Ref. Ref. 12). Finally, it is shown in Table II that the calculated positions of the crystal-field levels agree in most details with the spectroscopic observations. We therefore conclude that the present set of crystal-field parameters is trustworthy, as it accounts for an extensive number of observations in a consistent way.

The total Hamiltonian includes the crystal-field, the hyperfine, the Zeeman, and the classical dipole-dipole interactions. Finally, a nearest-neighbor Heisenberg exchange interaction is included:

$$
\begin{aligned}
\mathcal{H}= & \sum_{i}\left[\mathcal{H}_{\mathrm{CF}}\left(\mathbf{J}_{i}\right)+A \mathbf{J}_{i} \cdot \mathbf{I}_{i}-g \mu_{B} \mathbf{J}_{i} \cdot \mathbf{H}\right] \\
& -\frac{1}{2} \sum_{i j} \sum_{\alpha \beta} \mathcal{J}_{D} D_{\alpha \beta}(i j) J_{i \alpha} J_{j \beta}-\frac{1}{2} \sum_{i j}^{\mathrm{nn}} \mathcal{J}_{12} \mathbf{J}_{i} \cdot \mathbf{J}_{j}
\end{aligned}
$$

$\mathbf{J}_{i}$ and $\mathbf{I}_{i}$ are, respectively, the electronic angular momentum and the nuclear spin of the $i$ th ion, where $J=8$ with $g=5 / 4$ and $I=7 / 2$ for ${ }^{165}$ Ho. Hyperfine resonance ${ }^{12}$ and heatcapacity measurements ${ }^{24}$ show that the hyperfine coupling parameter is $A=3.36 \mu \mathrm{eV}$ as for the isolated ion. ${ }^{25}$ The heatcapacity measurements also indicate that the nuclear quadrupole coupling has the same magnitude as in the isolated ion, which coupling may therefore be safely neglected in the present calculations. The number of Ho ions per unit volume is $N=1.389 \times 10^{22} \mathrm{~cm}^{-3}$, and the dipole coupling parameter $\mathcal{J}_{D}=\left(g \mu_{B}\right)^{2} N=1.1654 \mu \mathrm{eV}$. The dipole sum is

$$
D_{\alpha \beta}(i j)=\frac{3\left(r_{i \alpha}-r_{j \alpha}\right)\left(r_{i \beta}-r_{j \beta}\right)-\left|\mathbf{r}_{i}-\mathbf{r}_{j}\right|^{2} \delta_{\alpha \beta}}{N\left|\mathbf{r}_{i}-\mathbf{r}_{j}\right|^{5}}
$$

and its Fourier transform in this case with four sublattices is calculated using the method of Bowden and Clark. ${ }^{26}$ At zero wave vector

$$
\begin{aligned}
& \mathcal{J}_{D} D_{a a}(\mathbf{0})=\mathcal{J}_{D}\left(\frac{4 \pi}{3}-0.83225\right)=3.912 \mu \mathrm{eV}, \\
& \mathcal{J}_{D} D_{c c}(\mathbf{0})=\mathcal{J}_{D}\left(\frac{4 \pi}{3}+1.66451\right)=6.821 \mu \mathrm{eV}
\end{aligned}
$$

The demagnetization field is subtracted from the applied field in the Zeeman term, when a uniform magnetization is present. This field is determined as the demagnetization factor times the relative magnetization times the maximum field $H_{d}^{0}=4 \pi M_{0}=4 \pi g \mu_{B} J N=16.19 \mathrm{kOe}$. For completeness, we add that the demagnetization field cancels out in the determination of the critical condition, no matter the shape of the individual domains in the ordered phase, ${ }^{18,27}$ in contrast to the suggestion by Chakraborty et al. ${ }^{17}$ This follows because in zero applied field (in the longitudinal direction) the crystal will always divide itself into domains so that the magnetic 
surface-charge density vanishes, or, phrased differently, the ordering is going to occur at wave vectors infinitesimally different from zero, not at $q \equiv 0$ but at the wave vectors where the paramagnetic excitation spectrum has its minimum value (see below).

The good description obtained for elevated temperatures or high energies does not completely settle the values of the crystal-field parameters, but the room left for variations is strongly limited. In the phase-diagram calculations presented below $B_{6}^{4}(s)$ is utilized as the variational parameter, since even a $20 \%$ change of this parameter only has slight consequences for the crystal-field level scheme. Except for this "fine-tuning" possibility offered by $B_{6}^{4}(s)$, the only free parameter in the Hamiltonian in Eq. (2) is the nearest-neighbor exchange interaction $\mathcal{J}_{12}$, and this parameter is small compared to $\mathcal{J}_{D} D_{c c}(\mathbf{0})$. The system is therefore well characterized from the outset, suggesting that it should be a good candidate for the testing of refined theories.

Bitko et al. produced a reasonable fit to the critical transverse field as a function of temperature within the MF approximation. ${ }^{6}$ They used the crystal-field parameters of Hansen et al. and $\mathcal{J}_{12}=-0.542 \mu \mathrm{eV}$ (in our notation). This MF model leads to the right $T_{C}$ at zero-field, but the critical field in the zero-temperature limit is only about $60 \%$ of the observed one. The inadequacy of the parameters of Hansen et al. was circumvented by introducing an effective $g$ factor for the transverse field $\left(g_{\perp}=0.59 \mathrm{~g}\right)$. This possibility for an adjustment of the crystal-field behavior is no longer acceptable, when using the present consistent set of crystal-field parameters. Even with an optimal choice of the parameters in Eq. (1) we have only been able to produce a MF model with a critical field at zero temperature, which is about $10 \%$ smaller than the observed one. It is possible to construct a MF model that reproduces the experimental results for the critical field, when it is larger than $20 \mathrm{kOe}$; however, this fit predicts the zero-field transition to lie between 1.8 and $1.85 \mathrm{~K}$ instead of at $1.53 \mathrm{~K}$, and it is also found that the RPA excitation energies are about $22 \%$ smaller than observed. These discrepancies indicate that it is important to include the fluctuations, and we shall see that the leading-order corrections to the MF model have the right sign, but do not explain all the differences.

\section{B. The $1 / z$ expansion and the phase diagram}

The effects of the fluctuations are included by performing a systematic high-density expansion ${ }^{3}$ of the two-site Green's function, i.e., of the $\tau$-ordered ensemble average

$$
G\left(i j, \tau_{1}-\tau_{2}\right)=-\left\langle T_{\tau} \widetilde{J}_{i z}\left(\tau_{1}\right) \widetilde{J}_{j z}\left(\tau_{2}\right)\right\rangle
$$

where $\tilde{J}_{i z}=J_{i z}-\left\langle J_{i z}\right\rangle$. This expansion is particularly well suited for a system like the present one with long-range interaction, implying a large effective number $z$ of coupled neighbors. To zeroth order, the fluctuations are neglected and the theory is identical with the RPA theory. To first order the theory includes the effects of the fluctuations in the surroundings of each single site. These single-site fluctuations may be accounted for in a self-consistent manner since the fluctuating surroundings, to first order in $1 / z$, constitute an "effective medium," which is common for every single site. ${ }^{15,16}$ The Fourier transform of the Green's function is $G\left(\mathbf{q}, i \omega_{n}\right)$, where $\omega_{n}=2 \pi n k_{B} T / \hbar$ is the Matsubara frequency, in terms of which the single-site Green's function is

$$
G\left(i \omega_{n}\right) \equiv G\left(j j, i \omega_{n}\right)=\frac{1}{N} \sum_{\mathbf{q}} G\left(\mathbf{q}, i \omega_{n}\right) .
$$

The final Green's function is then determined selfconsistently in terms of $G\left(i \omega_{n}\right)$ and the effective-medium coupling $K\left(i \omega_{n}\right)$ :

$$
G\left(\mathbf{q}, i \omega_{n}\right)=\frac{G\left(i \omega_{n}\right)}{1+\left\{\mathcal{J}(\mathbf{q})-K\left(i \omega_{n}\right)\right\} G\left(i \omega_{n}\right)} .
$$

$\mathcal{J}(\mathbf{q})$ is the Fourier transform of the total two-ion coupling (the eigenvalues of the $4 \times 4$ coupling matrix), and

$$
K\left(i \omega_{n}\right)=\frac{1}{N} \sum_{\mathbf{q}} \mathcal{J}(\mathbf{q}) \frac{G\left(\mathbf{q}, i \omega_{n}\right)}{G\left(i \omega_{n}\right)}
$$

is the sum of all chain diagrams, which start and end at the same site without crossing this site in between. The singlesite Green's function itself is determined by a Dyson equation which includes the second- and fourth-order cumulants

$$
G\left(i \omega_{n}\right)=\frac{G_{0}\left(i \omega_{n}\right)}{1+K\left(i \omega_{n}\right) G_{0}\left(i \omega_{n}\right)+\Sigma\left(i \omega_{n}\right)} .
$$

$G_{0}\left(i \omega_{n}\right)$ is the noninteracting Green's function and $\Sigma\left(i \omega_{n}\right)$ is the self-energy deriving from the fourth-order cumulant. The single-site dynamics is influenced by the coupling to the surrounding effective medium, which, in turn, determines the properties of the effective medium.

The effective-medium theory to first order in $1 / z$ has been applied previously to the similar system $\mathrm{HoF}_{3} \cdot{ }^{18-20}$ The only difference of importance between the two systems is that $\mathrm{HoF}_{3}$ is a singlet-singlet system also at zero field, allowing the hyperfine interaction to be included by second-order perturbation theory. ${ }^{16}$ This approximation is also acceptable in the present system, whenever the splitting of the ground-state doublet is somewhat larger than the hyperfine splitting of $1.5 \mathrm{~K}$. However, in order to account for the degenerate case at low fields, the hyperfine interaction in $\mathrm{HoLiF}_{4}$ has been included via an exact diagonalization of the $(2 J+1) \times(2 I$ $+1)$ single-ion Hamiltonian as also done by Bitko et al. ${ }^{6}$ Except for this modification, the theoretical results presented below are derived using the theory developed for the case of $\mathrm{HoF}_{3}$, and we refer to Ref. 18 for a detailed presentation of the theory and for the result obtained for the self-energy $\Sigma\left(i \omega_{n}\right)$ in Eq. (8). The renormalization of the moment along the transverse field should be of no importance, as the meanfield component of the effective field is only about $5 \%$ of the applied field at maximum. The mixing of the ground-state doublet with the higher-lying excited states induced by the transverse field, also implies that the $c c$ component of the susceptibility is affected by transitions between the doublet and the other states. Those contributions are included in the noninteracting susceptibility, but are very small (less than $1 \%$ at zero frequency at maximum field) implying that the 
singlet-singlet determination of the renormalization effects is acceptable.

The theoretical result for the static susceptibility is similar to the MF result except that the $c c$ component is divided by a renormalization factor $1+\Sigma(0)$. At the critical point at zero field, the self-energy is

$$
\Sigma_{c}(0 ; H=0)=\frac{1}{N} \sum_{\mathbf{q}} \frac{\mathcal{J}(\mathbf{q})}{\mathcal{J}(\mathbf{0})-\mathcal{J}(\mathbf{q})} .
$$

This parameter depends weakly on the value assumed for $\mathcal{J}_{12}$, and in the final fit it is calculated to be 0.3004 (for comparison, this value is 0.3447 in a fcc lattice with a nearest-neighbor coupling). As the energy gap opens up due to the application of a transverse field, the critical value $\Sigma_{c}(0)$ decreases roughly linearly with temperature. In the final fit $\Sigma_{c}(0)=0.0932$ at $T=0.31 \mathrm{~K}$ and $H=43.0 \mathrm{kOe}$, and is 0.0493 in the zero-temperature limit at the field $H$ $=52.9 \mathrm{kOe}$. The value of the Heisenberg exchange parameter in the final fit is

$$
\mathcal{J}_{12}=-0.1 \mu \mathrm{eV} \text {, }
$$

about a factor of 5 smaller than in the MF model. The effective coupling at zero wave vector is $\mathcal{J}(\mathbf{0})=\mathcal{J}_{D} D_{c c}(\mathbf{0})+4 \mathcal{J}_{12}$ $=6.421 \mu \mathrm{eV}$. The calculated phase diagram is compared with the experimental one in Fig. 1.

The unusual upturn in $H_{c}$ below $400 \mathrm{mK}$ in the phase diagram was explained by alignment of the Ho nuclear moments through the hyperfine coupling by Bitko et al. ${ }^{6}$ Corrections to phase diagrams due to hyperfine couplings have a long and venerable history, ${ }^{28}$ and were noted for the $\mathrm{Li} R \mathrm{FF}_{4}$ ( $R=$ rare earth) series over 20 years ago. ${ }^{29}$ Here, the hyperfine enhancement becomes important below $0.4 \mathrm{~K}$, and increases the critical field by about $25 \%$ in the zerotemperature limit.

The theory agrees with both sets of experimental data, when the transverse field lies between 20 and $40 \mathrm{kOe}$, and with the results of Bitko et al. below $0.4 \mathrm{~K}$. The present effective-medium phase diagram suffers from the same shortcoming as the MF result, namely, that the Curie temperature at zero field is higher than observed, $1.74 \mathrm{~K}$, or $14 \%$ above the experimental value. The discrepancy has been reduced but is still quite substantial. An alternative fit is shown by the dot-dashed line in Fig. 1, which is obtained by using $\mathcal{J}_{12}=-0.27 \mu \mathrm{eV}$ and a slightly different value of $B_{6}^{4}(s)$ $=0.75 \times 10^{-5} \mathrm{meV}$. This calculated phase line agrees with the experiments at zero field and with the results of Bitko et al. below $0.4 \mathrm{~K}$; however, the results at the intermediate fields differ significantly from the experimental ones. The basic difficulty with the interpretation of the experimental results is the very rapid variation of the transverse critical field, from 0 to $20 \mathrm{kOe}$ within an interval of less than $0.1 \mathrm{~K}$.

\section{The $1 / z$ expansion and the excitations}

We now report the $1 / z$ expansion results for the excitations, which was used to analyze the inelastic neutron scattering data reported previously. 8,30

The dominating role of the classical dipole-dipole interaction implies an extremely anisotropic behavior of the excita-

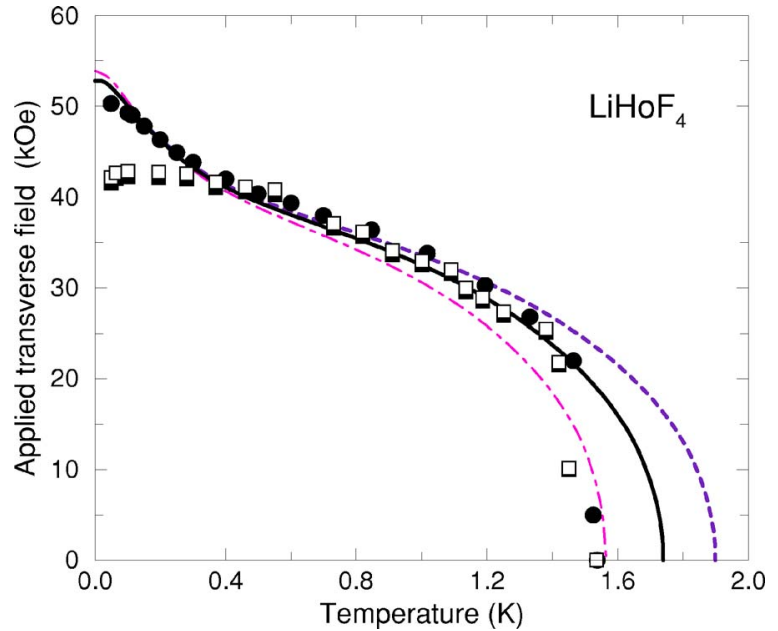

FIG. 1. (Color online) Phase diagram of $\mathrm{LiHoF}_{4}$ as a function of experimental temperature and field. The solid circles are the experimental results of Bitko et al. (Ref. 6) obtained on a spherical sample. In the neutron experiments (squares), the thermometer was located at the mixing chamber, neglecting any temperature gradient to the sample. Comparing to the results of Bitko et al., we determined that all data, including the inelastic data (Ref. 8), at recorded lower nominal temperatures were actually recorded at the same $T$ $=0.31 \pm 0.02 \mathrm{~K}$. The phase boundary is determined from the magnetic component of the neutron scattering intensities at (200) and (101), whereas the open squares are the results when corrected for the difference between the demagnetization field in our sample (demagnetization factor of about 0.25 ) and a spherical one. The solid line is the $1 / z$ calculation with $\mathcal{J}_{12}=-0.1 \mu \mathrm{eV}$, which is compared (the dashed line) with the Monte Carlo prediction of Chakraborty et al. (Ref. 17) scaled to the present Hamiltonian. The dot-dashed line is the $1 / z$ result with $\mathcal{J}_{12}=-0.27 \mu \mathrm{eV}$.

tion spectrum in the long-wavelength limit, with the lowestenergy excitation located at points where the resulting wave vector is nearly zero but perpendicular to the $c$ axis. Figure 2 presents this lowest excitation energy as a function of the applied transverse field. Both to zeroth (RPA) and first order in the high-density $1 / z$ expansion, the simple singlet-singlet system should show a soft-mode transition at the critical field. This is not the case with the present system because the low-frequency dynamics are modified by the hyperfine interaction, which effectively leads to an extra pole in the noninteracting susceptibility at about $0.01 \mathrm{meV}$. The consequence is that there is a gap in the crystal-field excitation spectrum also close to the phase transition, while the critical fluctuations at low frequencies derive from the low-frequency pole. To first order in $1 / z$ the dynamic susceptibility is similar to the RPA result except that the noninteracting $c c$ component is divided by $1+\Sigma(\omega)$. The application of the expression derived for $\Sigma(\omega)$ in Ref. 18 leads to the calculated results that are displayed in Fig. 2, where they are compared with the experimental results. The theory describes the qualitative behavior of the excitation energy as a function of field; however, actual agreement is only obtained when scaling the calculated energies by a factor of about 1.15.

Our theory can also provide the dispersion of the excitations as a function of momentum $Q$ and the scattering inten- 


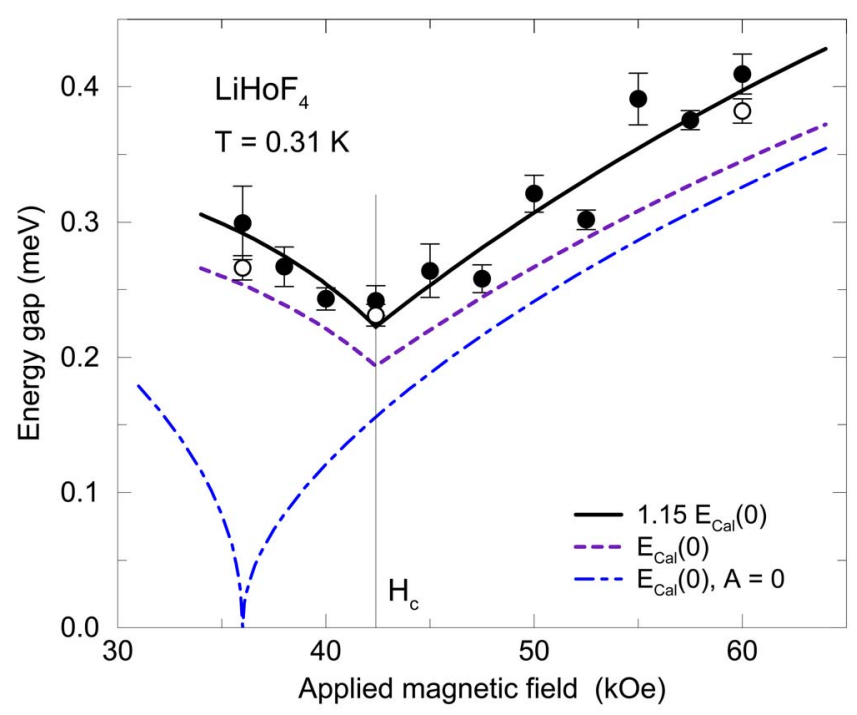

FIG. 2. (Color online) Field dependence at $T=0.31 \mathrm{~K}$ of the lowest-energy excitation in $\mathrm{LiHoF}_{4}$ at $Q=(1+\epsilon, 0,1)$ (closed circles) and at $Q=(1.9,0,0)$ (open circles). The dashed line shows the calculated results, and the solid the calculated energies scaled with a factor of 1.15. The dot-dashed line shows the soft-mode behavior expected, if the hyperfine interaction is neglected.

sity. From the neutron scattering data, the dispersion and scattering intensities were extracted along various symmetry directions in the Brillouin zone at the critical field, $42.4 \mathrm{kOe}$, and on each side of the critical field, at 36 and $60 \mathrm{kOe}$. As illustrated in Fig. 3, the measured dispersion is well described by our theory. More experimental results are presented in Refs. 8 and 30. The calculated excitation energies are systematically smaller than the measured ones, but once again the agreement becomes acceptable if all the calculated energies are scaled by a factor 1.15 , as shown in Fig. 3 for

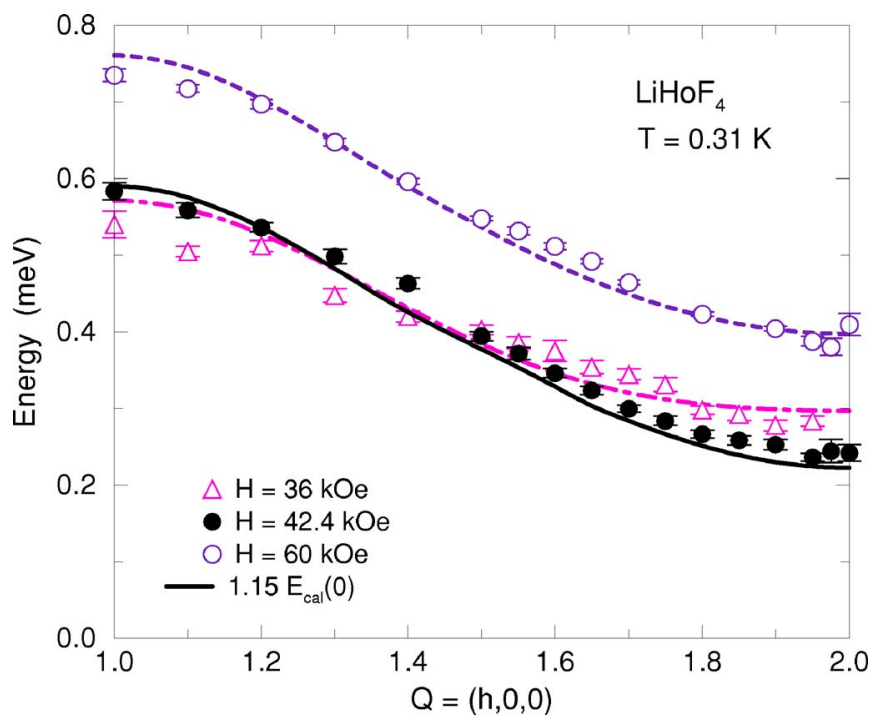

FIG. 3. (Color online) Dispersion along $\mathbf{Q}=(h, 0,0)$ at the three different values of the transverse field at $T=0.31 \mathrm{~K}$. The lines are the calculated energies scaled by a factor of 1.15 , for the fields 36 (dot-dashed), 42.4 (solid), and $60 \mathrm{kOe}$ (dashed). the case of $\mathbf{Q}$ along [100]. The comparison of the calculated and measured scattering intensities involves a single overall scale parameter. The intensities are roughly proportional to the squared matrix element divided by the excitation energies, and they decline rapidly when the ordered moment evolves. The relative variation of the intensities at $42.4 \mathrm{kOe}$ is well described by the theory (see Fig. 3 in Ref. 8).

\section{DISCUSSION}

The crystal-field Hamiltonian for the $\mathrm{Ho}$ ions in $\mathrm{LiHoF}_{4}$ is well characterized by the high-temperature susceptibility measurements and the spectroscopic investigations of the crystal-field level scheme. The most important magnetic interaction between the Ho ions is the classical dipole-dipole interaction, which is calculable from first principles. The only adjustable part left in the Hamiltonian is the exchange interaction, probably dominated by the interaction $\mathcal{J}_{12}$ between nearest neighbors. The renormalization of the effective MF-RPA parameters due to fluctuations depends on field, temperature, and frequency. The inclusion of these modifications, as predicted by the effective-medium theory to first order in the density $1 / z$, clearly improves the comparison between theory and observations. When considering that most parameters in the Hamiltonian are fixed in advance, the theoretical description presented of the phase diagram and of the low temperature excitation spectrum is highly satisfactory. However, the discrepancies left are sufficiently large to indicate that either the effective-medium theory is inaccurate or the modeling of the system is incomplete in some other way.

The magnetic phase diagram of $\mathrm{LiHoF}_{4}$ has recently been calculated by Chakraborty et al. using a quantum Monte Carlo method, a method that is expected to allow for an accurate determination of the critical field, ${ }^{17}$ accounting particularly for the mixed (ferro- and antiferromagnetic) nature and intermediate range of the dipolar interaction. A comparison of the results derived from these calculations with the result of the effective-medium $1 / z$ theory presented in Fig. 1 shows that the ground state properties predicted by the present theory are trustworthy whenever the splitting of the doublet state is appreciable, i.e., when the transverse field is larger than about $20 \mathrm{kOe}$. However, the $1 / z$ theory is found to overestimate the reduction of the transition temperature caused by the fluctuations, when the field is small. This comparison is very similar to the one shown in Fig. 5 of Ref. 15, where the $1 / z$ theory is compared with high-temperature expansion results in the case of $S=1$. Assuming the Monte Carlo calculation predicts the correct phase diagram, the corrected phase line, in the case of $\mathcal{J}_{12}=-0.1 \mu \mathrm{eV}$, would be close to the solid line at temperatures below $1.2 \mathrm{~K}$, but the transition temperature at zero field would occur at $1.90 \mathrm{~K}$ rather than $1.74 \mathrm{~K}$ as predicted by the $1 / z$ theory.

The ignorance of the direction of the $x$ axis in the crystalfield Hamiltonian is of no importance for the comparison between theory and experiments. The critical field is predicted to vary by about $2.8 \mathrm{kOe}$ at $0.31 \mathrm{~K}$ depending on the choice of angle $x$ makes with [100], but the calculated properties, both the phase diagram and the excitation spectrum, 
are practically unchanged if this field variation is counterbalanced by a small adjustment of $B_{6}^{4}(s)$. For instance, if assuming the angle to be $-11^{\circ}$-in the domain where $B_{6}^{4}(s)>0$ - as predicted by the point charge model, then the change from 0 to $-11^{\circ}$ is compensated for by a $4 \%$ increase of $B_{6}^{4}(s)$.

The scale factor of about 1.15 in the comparison between the calculated and observed excitation energies indicates that the splitting of the crystal-field doublet state $\Delta$ and the squared matrix element of $J_{c}$ between the doublet states is a factor of 1.15 larger in the system than predicted by the model. At the critical field, the ratio between the squared matrix element of $J_{c}$ and $\Delta$ (half the susceptibility) is fixed by the value of the field. The simple transverse-field Ising model predicts $\Delta=2 k_{B} T_{C}(0)$, where $T_{C}(0)$ is the value of the Curie temperature at zero field. In the present system, the $1 / z$ theory determines the renormalization of the mean-field relation to be a factor of 1.19 (at $0.31 \mathrm{~K}$ ), and the mixing of the higher-lying levels into the ground state doublet due to the transverse field increases $\Delta$ by $5-10 \%$. Hence, $\Delta$ is about $2.6 k_{B} T_{C}(0)=0.39 \mathrm{meV}$ at the critical field at $0.31 \mathrm{~K}$. The energy of the lowest-lying mode at this field is $E_{c}(0)$ $\simeq \Delta \sqrt{1-\chi_{J}(0) / \chi(0)}$, where $\chi(0)$ is the noninteracting susceptibility at zero frequency and $\chi_{J}(0)$ is the same quantity when the nuclear contribution is neglected. At $0.31 \mathrm{~K}$, the ratio $\chi_{J}(0) / \chi(0) \simeq 0.77$ and the estimate leads to $E_{c}(0)$ $\simeq 0.19 \mathrm{meV}$ in accordance with the calculated value of $0.193 \mathrm{meV}$. The only way a modification of the crystal-field parameters may affect the excitation energies, under the condition that the critical-field phase line stays fixed, is through the $5-10 \%$ increase of $\Delta$ produced by the field-induced mixing of the states. To account for the scale factor this modification should instead be $20-25 \%$, which is unattainable. We have tried many different sets of crystal-field parameters, relaxing on the comparison with the susceptibility and spectral measurements, without being able to produce any better fit to the excitation energies.

The need for the excitation-energy scale factor of 1.15 is possibly related to the steep growth of the critical field shown by the system below $T_{C}(0)$. The steep rise of the critical field is not reproducible by the present model, and introducing the corrections to the $1 / z$ theory, as indicated by the Monte Carlo calculations, would worsen the comparison. This discrepancy may reflect the neglect of large domain walls and shape effects in both calculations. The walls can roughen as the phase boundary is approached and so, given the long-range nature of the dipole interaction, have the potential to produce renormalizations of the observed static and dynamic behavior. Particularly interesting is the possibility that these effects differ in the low-temperature quantum regime and in the higher-temperature classical domain. Indeed Brooke et al. ${ }^{10}$ already showed for the dilute ferromagnet $\mathrm{LiHo}_{x} \mathrm{Y}_{1-x} \mathrm{~F}_{4}$ with $x=0.44$ that the domain wall dynamics undergo a classical-quantum crossover on cooling. Another point to make is that our theory is a mean-field-like approach, and therefore cannot generate the celebrated logarithmic terms associated with the classical phase transition in $\mathrm{LiHoF}_{4}$ (Ref. 31) and $\mathrm{LiTbF}_{4} \cdot{ }^{32,33}$ Such corrections (likely to be missed in Quantum Monte Carlo calculations restricted to finite size samples) will also manifest themselves in the phase boundary as the transverse field vanishes and the system becomes classical, and we look forward to appropriate renormalization group calculations.

The domain walls may be important for the critical dynamics at low frequencies, but have no direct consequences on the high-frequency excitations studied here. Both our effective-medium theory and the quantum Monte Carlo calculations neglect magnetoelastic quadrupole interactions. The most significant term of this type is $-B_{21}\left[O_{2}^{1}(c)\left\langle O_{2}^{1}(c)\right\rangle\right.$ $\left.+O_{2}^{1}(s)\left\langle O_{2}^{1}(s)\right\rangle\right]$ and introducing $B_{21}=0.55 \times 10^{-4} \mathrm{meV}$, and an appropriate increase of $B_{6}^{4}(s)$ by $15 \%$, then the excitation of lowest energy is shifted upward by the required $15 \%$, though the dispersion band width is slightly (3\%) decreased. A magnetoelastic coupling of this size agrees in order of magnitude with that predicted by a point-charge model. Its inclusion in the Hamiltonian would improve the overall description of the excitation spectrum; however, its effect on the critical field would only be a shift by about $1 \mathrm{kOe}$ at maximum, barely visible in Fig. 1. Therefore, since this extra degree of freedom in the Hamiltonian only allows a partial removal of the discrepancies and is not based on rigorous measurement or calculation of $B_{21}$, it is an open question whether it is significant or not. Additional observations, e.g., via high-resolution $\mathrm{x}$-ray diffraction, are required in order to justify a $B_{21}$ of the suggested magnitude, such as for instance an observation of a $\epsilon_{13}$ strain of the order of $10^{-4}$ at fields slightly below the critical field at the lowest temperatures.

Brooke et al.,${ }^{10}$ Bitko, ${ }^{7}$ and $\mathrm{Wu}$ et al. ${ }^{34}$ have presented the phase diagram of the magnetically diluted system $\mathrm{LiHo}_{x} \mathrm{Y}_{1-x} \mathrm{~F}_{4}$, with $x=0.46$ and 0.167 . The transition temperature at zero field scales with $x$ in accordance with the virtual-crystal approximation and with the coherent-potential approximation (CPA). However, in the zero-temperature limit, the critical field is observed to be about $17 \mathrm{kOe}$ when $x=0.44$, whereas the virtual-crystal MF model and the present model in combination with the CPA both predict that the critical field should be more than a factor of 2 larger (the nonlinearity of the dependence of the calculated $H_{c}$ on $x$ is due to the fact that the splitting of the ground state doublet has an expansion in external field which scales like $H^{2}$ ). The highly diluted system, $x=0.167$, shows a transition to a spinglass phase, ${ }^{5}$ but also the less diluted system with $x=0.44$ shows hysteresis and other glassy features. ${ }^{7}$ The steep rise of the critical field when $x=1$ may possibly be an effect of the domain walls; however, the factor-of-two difference between the two values of the critical field in the $x=0.44$ case indicates the presence of an additional modification of the lowfrequency dynamics of the system. The idea that the physics is that of a dense network of randomly pinned domain walls, as described by Brooke et al. for the $x=0.44$ sample, ${ }^{10}$ is in accord with this suggestion of a crucial missing ingredient in our model even in the limit of pure $\mathrm{LiHoF}_{4}$. It is well known that the effects of substitutional disorder are amplified in the quantum limit, ${ }^{35}$ especially because of internally generated random transverse fields. Very recent work ${ }^{36}$ which takes account of this as well as the hyperfine interactions is beginning to yield a quantitative theory for the diluted rare-earth fluorides. Another source of randomness (apart from that due to replacing Ho by $\mathrm{Y}$ ) is isotopic disorder due to the presence 
of two different Li isotopes. Their different zero-point motion might cause a site-random distortion of the crystal-field Hamiltonian, and isotope shifts in the optical transitions of dilute $\mathrm{LiHo}_{x} \mathrm{Y}_{1-x} \mathrm{~F}_{4}$ have been reported. ${ }^{37}$ We found that $T_{c}$ $=1.53 \pm 0.03$ in a crystal prepared exclusively with the ${ }^{7} \mathrm{Li}$ isotope, exactly the same as in natural $\mathrm{Li}$ samples. This result will be supplemented with a study of the critical field as a function of temperature.

\section{CONCLUSIONS}

We have presented a theory for the excitation spectrum in $\mathrm{LiHoF}_{4}$ and shown that it accounts well for the neutron scattering experiments reported previously. ${ }^{8}$ The principal feature of both experiment and calculation is a strong magnetic mode which softens as the quantum phase transition is approached. Upon lowering the field from the paramagnetic phase, the mode follows the predictions for the ordinary transverse-field Ising model until its energy becomes comparable to the hyperfine interaction strength, at which point new and much lower-energy modes appear and the ferromagnetic state seems to emerge prematurely. Thus, the softening to zero of this most visible mode is forestalled. Our detailed effective-medium calculations give a quantitative account of all magnetic data for $\mathrm{LiHoF}_{4}$-including the excitations and the phase diagram. Our study provides a quantitative understanding of the excitations near the quantum critical point of a model experimental system. In addition, and perhaps more important, it represents, as described in our previous account of this work, ${ }^{8}$ an excellent example of how a nuclear spin bath affects quantum dynamics in the limit of an infinite number of degrees of freedom. Our work thus complements the efforts on decoherence in magnetic molecules, ${ }^{38}$ where there is only a finite number of degrees of freedom.

\section{ACKNOWLEDGMENTS}

We thank M. Schechter for stimulating discussions and G. McIntyre for assistance during complementing measurements on the D10 diffractometer at Institut Laue Langevin, France. Work at the University of Chicago was supported by NSF Materials Research Science and Engineering Centers Grant No. DMR-0213745 and U.S. Department of Energy Grant No. DE-FG02-99ER45789. Work in London was supported by the Wolfson-Royal Society Program and the Basic Technologies program of the U.K. Research Councils.
${ }^{1}$ S. Sachdev, Phys. World 12 (4), 33 (1999); Quantum Phase Transitions (Cambridge University Press, Cambridge, U.K., 1999).

${ }^{2}$ P. G. de Gennes, Solid State Commun. 1, 132 (1963).

${ }^{3}$ R. B. Stinchcombe, J. Phys. Chem. Ref. Data Suppl. 6, 2459 (1973); 6, 2484 (1973).

${ }^{4}$ R. J. Elliott, P. Pfeuty, and C. Wood, Phys. Rev. Lett. 25, 443 (1970).

${ }^{5}$ T. F. Rosenbaum, W. Wu, B. Ellman, J. Yang, G. Aeppli, and D. H. Reich, J. Appl. Phys. 70, 5946 (1991).

${ }^{6}$ D. Bitko, T. F. Rosenbaum, and G. Aeppli, Phys. Rev. Lett. 77, 940 (1996).

${ }^{7}$ D. Bitko, Ph.D. Thesis, University of Chicago, Illinois, 1997.

${ }^{8}$ H. M. Rønnow, R. Parthasarathy, J. Jensen, G. Aeppli, T. F. Rosenbaum, and D. F. McMorrow, Science 308, 389 (2005).

${ }^{9}$ R. Giraud, W. Wernsdorfer, A. M. Tkachuk, D. Mailly, and B. Barbara, Phys. Rev. Lett. 87, 057203 (2001).

${ }^{10}$ J. Brooke, T. F. Rosenbaum, and G. Aeppli, Nature (London) 413, 610 (2001); S. Ghosh, T. F. Rosenbaum, G. Aeppli, and S. N. Coppersmith, ibid. 425, 48 (2003); J. Brooke, D. Bitko, T. F. Rosenbaum, and G. Aeppli, Science 284, 779 (1999); S. Ghosh, R. Parthasarathy, T. F. Rosenbaum, and G. Aeppli, ibid. 296, 2195 (2002).

${ }^{11}$ P. E. Hansen, T. Johansson, and R. Nevald, Phys. Rev. B 12, 5315 (1975).

${ }^{12}$ J. Magarinino, J. Tuchendler, P. Beauvillain, and I. Laursen, Phys. Rev. B 21, 18 (1980).

${ }^{13}$ H. P. Christensen, Phys. Rev. B 19, 6564 (1979).

${ }^{14}$ S. Salaün, M. T. Fornoni, A. Bulou, M. Rousseau, P. Simon, and J. Y. Gesland, J. Phys.: Condens. Matter 9, 6941 (1997).

${ }^{15}$ J. Jensen, J. Phys. C 17, 5367 (1984).

${ }^{16} \mathrm{~J}$. Jensen and A. R. Mackintosh, Rare Earth Magnetism: Struc- tures and Excitations (Clarendon Press, Oxford, 1991).

${ }^{17}$ P. B. Chakraborty, P. Henelius, H. Kjønsberg, A. W. Sandvik, and S. M. Girvin, Phys. Rev. B 70, 144411 (2004).

${ }^{18}$ J. Jensen, Phys. Rev. B 49, 11833 (1994).

${ }^{19}$ M. J. M. Leask, M. R. Wells, R. C. C. Ward, S. M. Hayden, and J. Jensen, J. Phys.: Condens. Matter 6, 505 (1994).

${ }^{20}$ A. P. Ramirez and J. Jensen, J. Phys.: Condens. Matter 6, L215 (1994).

${ }^{21}$ K. Kjaer, J. Als-Nielsen, I. Laursen, and F. Krebs Larsen, J. Phys.: Condens. Matter 1, 5743 (1989).

${ }^{22}$ Sh. N. Gifeisman, A. M. Tkachuk, and V. V. Prizmak, Opt. Spectrosc. 44, 68 (1978).

${ }^{23} \mathrm{C}$. Görller-Walrand and K. Binnemans, in Handbook on the Physics and Chemistry of Rare Earths, edited by K. A. Gschneidner, Jr. and L. Eyring (North-Holland, Amsterdam, 1996), Vol. 23, p. 121.

${ }^{24}$ G. Mennenga, L. J. de Jongh, and W. J. Huiskamp, J. Magn. Magn. Mater. 44, 59 (1984).

${ }^{25}$ B. Bleaney, in Magnetic Properties of Rare Earth Metals, edited by R. J. Elliott (Plenum Press, London, 1972), p. 383.

${ }^{26}$ G. J. Bowden and R. G. Clark, J. Phys. C 14, L827 (1981).

${ }^{27}$ F. Keffer, in Ferromagnetismus, edited by H. P. J. Wijn, Handbuch der Physik Vol. XVIII/2 (Springer Verlag, Berlin, 1966), p. 1.

${ }^{28}$ K. Andres, Phys. Rev. B 7, 4295 (1973).

${ }^{29}$ R. W. Youngblood, G. Aeppli, J. D. Axe, and J. A. Griffin, Phys. Rev. Lett. 49, 1724 (1982).

${ }^{30}$ For details, see H. M. Rønnow, Ph.D. Thesis, Ris $\varnothing$ National Laboratory, Denmark, 2000.

${ }^{31}$ J. A. Griffin, M. Huster, and R. J. Folweiler, Phys. Rev. B 22, 4370 (1980).

${ }^{32}$ J. Als-Nielsen, L. M. Holmes, and H. J. Guggenheim, Phys. Rev. 
Lett. 32, 610 (1974).

${ }^{33}$ G. Ahlers, A. Kornblitt, and H. J. Guggenheim, Phys. Rev. Lett. 34, 1227 (1975).

${ }^{34}$ W. Wu, D. Bitko, T. F. Rosenbaum, and G. Aeppli, Phys. Rev. Lett. 71, 1919 (1993).

${ }^{35}$ E. R. Mucciolo, A. H. Castro Neto, and C. Chamon, Phys. Rev. B 69, 214424 (2004).
${ }^{36}$ M. Schechter and P. C. E. Stamp, Phys. Rev. Lett. 95, 267208 (2005).

${ }^{37}$ N. I. Agladze, M. N. Popova, G. N. Zhizhin, V. J. Egorov, and M. A. Petrova, Phys. Rev. Lett. 66, 477 (1991).

${ }^{38}$ N. V. Prokof'ev and P. C. E. Stamp, Rep. Prog. Phys. 63, 669 (2000). 